\title{
Expression of $\mathrm{miR}-150$ and $\mathrm{miR}-3940-5 p$ is reduced in non-small cell lung carcinoma and correlates with clinicopathological features
}

\author{
YIFENG SUN $^{1 *}$, BO SU ${ }^{2 *}$, PENG ZHANG ${ }^{1 *}$, HUIKANG XIE ${ }^{3}$, HUI ZHENG ${ }^{1}$, YONGJIE XU ${ }^{1}$, \\ QIAOLING DU ${ }^{4}$, HUAN ZENG $^{3}, \mathrm{XIAO} \mathrm{ZHOU}^{1}, \mathrm{CHANG} \mathrm{CHEN}^{1}$ and WEN GAO ${ }^{5}$ \\ Departments of ${ }^{1}$ Thoracic Surgery, ${ }^{2}$ Central Laboratory, and ${ }^{3}$ Pathology, Shanghai Pulmonary Hospital, \\ Tongji University School of Medicine, Shanghai 200433; ${ }^{4}$ Department of Gynaecology and Obstetrics, \\ Shanghai First Maternity and Infant Health Hospital, Tongji University School of Medicine, Shanghai 200126; \\ ${ }^{5}$ Department of Thoracic Surgery, Shanghai Chest Hospital Affiliated with \\ Shanghai Jiaotong University, Shanghai 200030, P.R. China
}

Received October 2, 2012; Accepted November 9, 2012

DOI: $10.3892 /$ or.2012.2152

\begin{abstract}
The present study investigated the expression of miR-150 and miR-3940-5p in non-small cell lung carcinoma (NSCLC) and its relationship with clinicopathologic features. Samples included tumor, tumor-adjacent and normal lung parenchyma tissues from 90 NSCLC patients and 17 cases of embryonic lung cDNA. The expression levels of miR-150, miR-18b-5p, miR-643 and miR-3940-5p were detected by real-time PCR; p53, EGFR, Kras and Ki-67 expression in tumor tissues was determined by immunohistochemistry. p53 mRNA expression levels in NSCLC were examined by SYBR-Green real-time PCR. The relationship between the four miRNAs and clinicopathologic features of 90 cases was analyzed. The expression of miR-150 and miR-3940-5p was significantly downregulated in tumor tissues and embryonic lung tissues compared to normal lung tissues. The expression of miR-150 and miR-3940-5p in tumor tissues was also lower than that in the matched tumor-adjacent tissues. miR-150 was downregulated preferentially in subgroups of patients with a tumor diameter more than or equal to $3 \mathrm{~cm}$, in smokers and in stage III and IV tumors. Specifically, miR-150 and
\end{abstract}

Correspondence to: Dr Wen Gao, Department of Thoracic Surgery, Shanghai Chest Hospital Affiliated with Shanghai Jiaotong University, No. 241 Huaihaixi Road, Shanghai 200030, P.R. China E-mail: gaowen5921@yahoo.cn

Dr Chang Chen, Department of Thoracic Surgery, Shanghai Pulmonary Hospital, Tongji University School of Medicine, No. 507 Zhengmin Road, Shanghai 200433, P.R. China

E-mail: changchenc@hotmail.com

*Contributed equally

Key words: microRNAs, clinicopathology, non-small cell lung carcinoma, quantitative RT-PCR
miR-3940-5p expression was decreased in nuclear cell proliferation antigen Ki-67-positive NSCLC cases. miR-150 and miR-3940-5p were found to be significantly downregulated in p53 IHC-positive NSCLC cases and were negatively correlated with p53 mRNA. Reduced miR-150 and miR-3940-5p expression in tumor tissues and embryonic lung tissues suggests that these miRs may be involved in the tumorigenesis or de-differentiation of NSCLC. Due to this associaton with the Ki-67 proliferation index in NSCLC, downregulation of miR-150 and miR-3940-5p may contribute to tumor growth and proliferation. miR-150 and miR-3940-5p may affect p53 expression through a direct or indirect pathway.

\section{Introduction}

microRNAs (miRNAs) are a class of non-coding RNA genes whose final product is a 22-nt functional RNA molecule. They play important roles in the regulation of target genes by binding to complementary regions of messenger transcripts to repress translation or regulate degradation. This ultimately leads to repression of protein translation and downregulation of protein expression $(1,2)$. Deregulation of miRNAs is emerging as an important area of study in carcinogenesis since their regulatory capabilities can drastically influence cell physiology (3). Current data indicate that every tumor type analyzed by miRNA profiling shows differential expression of miRNAs when compared to normal tissues, therefore, differential expression of miRNAs may have diagnostic significance and prognostic value (4).

Lung cancer is the leading cause of cancer-associated mortality and is responsible for more deaths than the next three most common tumors combined (breast, prostate and colon) (5). Even for patients in the earliest stage of the disease (stage IA), the 5-year survival rate is only $73 \%$ after surgical resection (6). Non-small cell lung cancer (NSCLC) accounts for $\sim 87 \%$ of lung cancer cases (7). Recently, several studies have reported that alterations in miRNA expression 
are directly involved in the tumorigenesis of lung cancer and suggest that tissue miRNA profiling can be used for diagnosis or predicting the prognosis of lung cancer (8-10).

Our previous miRNA microarray analysis revealed that miRNA-150, miR-18b-5p, miR-643 and miR-3940-5p were downregulated in recurrent tumors when compared to primary tumors in NSCLC. To confirm the possible role of these miRNAs in NSCLC, we investigated the expression of miRNA-150, miR-18b-5p, miR-643 and miR-3940-5p in normal, tumor-adjacent and tumor tissues in 90 NSCLC patients. We also compared the expression levels with clinicopathological features and examined the expression level in embryonic lung cells. Our aim was to investigate the association of miRNA-150, miR-18b-5p, miR-643 and miR-3940-5p expression levels with clinicopathologic features and increase our understanding of the miRNA signature in NSCLC. The findings may suggest a potential diagnostic or prognostic strategy for targeting specific miRNAs.

\section{Materials and methods}

Approval for the present study was obtained from the ethics committees of the participating hospitals.

Patients and samples. Fresh frozen specimens from 90 cases of NSCLC used in this study were obtained from Shanghai Pulmonary Hospital between December 2010 and September 2011. The patients had not received adjuvant chemotherapy before surgery. Three samples, which included normal (N), tumor-adjacent normal (TN) and tumor (T) tissue, were obtained from excision specimens for each lung cancer patient. Tumor-adjacent samples were dissected $2 \mathrm{~cm}$ from the tumor and included as little contamination of the cancerous tissue as possible. Normal tissues consisted of the dissected pulmonary tissue at a distance of least $5 \mathrm{~cm}$ from the tumor. Samples were freshly resected after lung tissue excision and immediately frozen in liquid nitrogen for subsequent total RNA extraction. Tumors were classified according to the TNM staging for NSCLC (International Association for the Study of Lung Cancer, IASLC, 2009). The histological type, tumor size and pathologic differentiation were precisely diagnosed through routine cytological and histological examinations supplemented by histochemical and immunohistochemical assays. Seventeen embryonic lung tissue cDNA samples were kindly provided by Shanghai First Maternity and Infant Health Hospital.

Total RNA extraction and real-time PCR quantification of miRNAs. RNA was extracted from frozen tissue samples using a miRNeasy Mini kit (Qiagen GmbH, Hilden, Germany) following the manufacturer's instructions. cDNA was reverse transcribed from total RNA samples using the Qiagen miScript Reverse Transcription kit (Qiagen), which can polyadenylate at the $3^{\prime}$ terminus of miRNAs and reverse transcribe by a oligo-dT primer with a universal tag. All RT reactions were carried out in a $10-\mu l$ volume, starting with $1000 \mathrm{ng}$ of total RNA. The reaction mixture was initially heated to $37^{\circ} \mathrm{C}$ for $60 \mathrm{~min}$ and at $95^{\circ} \mathrm{C}$ for $5 \mathrm{~min}$. PCR products were amplified from cDNA samples using the Qiagen miScript SYBR-Green PCR kit with specific miRNA primers and miScript Universal Primer. Specific primer assays for each microRNA were obtained from Qiagen (order no. 2591821): hs-miR-150 (lot no. 102594470); hs-miR-18b-5p (lot no.1 02594478); hs-miR-643 (lot no. 102594477); hs-miR-3940-5p (lot no. 102594472); and Hs-RUN6-2-1 (lot no. 102594473).

miRNA PCR quantification was performed using the ABI PRISM 7500 Real-Time PCR system. The assay plates were initially heated to $95^{\circ} \mathrm{C}$ for $15 \mathrm{~min}$, followed by 40 cycles of $94^{\circ} \mathrm{C}$ for $15 \mathrm{sec}$ and $55^{\circ} \mathrm{C}$ for $30 \mathrm{sec}$. qPCR for each sample was performed in duplicate. The $-\Delta \Delta \mathrm{Ct}$ method was used to analyze the relative quantitative expression levels of miRNAs with snRNA U6 as an internal control gene. TP53/p53 was amplified from 60 cases of paired tumor and normal samples by SYBR-Green real-time PCR. TP53/p53 forward primer was TCAACAAGATGTTTTGCCAACTG; reverse primer, ATG TGCTGTGACTGCTTGTAGATG. cDNA was amplified using the following steps: an initial 3-min denaturation at $95^{\circ} \mathrm{C}$, followed by 40 cycles of $94^{\circ} \mathrm{C}$ for $5 \mathrm{sec}, 61^{\circ} \mathrm{C}$ for $34 \mathrm{sec}$ and a final melting curve step.

Immunohistochemistry of p53, EGFR, Kras and Ki-67. Immunohistochemistry was routinely performed to determine p53, EGFR, Kras and Ki-67 in the formalin-fixed paraffinembedded surgical specimens. Anti-p53 (clone DO-7; Dako, Glostrup, Denmark), anti-EGFR (clone EGFR.25; Gene Tech, Shanghai, China), anti-Kras (clone 4D10; Gene Tech), and antiKi-67 (clone MIB-1; Dako) monoclonal antibodies were used as primary Abs. A Ventana NexES immunohistochemistry kit (Ventana Medical Systems) was used for staining. Specimens were considered 'positive' when $>5 \%$ of tumor cells within the section exhibited positive nuclear staining for Ki-67 (11), positive nuclear staining for Kras, positive cytoplasmic or nuclear staining for p53 and positive membranous staining for EGFR. Immunohistochemical staining in surgical specimens was independently assessed by two pathologists.

Statistical analysis. Data are expressed as medians (IQR interquartile range). Statistical differences in miRNA expression between two groups were calculated using the non-parametric Wilcoxon Mann-Whitney test and a test for several independent samples used the Kruskal-Wallis $H$ test. A paired t-test was used for paired samples. Pearson's correlation was used to evaluate the correlation coefficient of the two groups. A Chi-square test was used to compare proportional data. The statistical analysis was performed using SPSS 17.0 software (SPSS Inc., Chicago, IL). The level of significance was defined as a $\mathrm{P}$-value of $<0.05$.

\section{Results}

General information. Ninety NSCLC cases were enrolled and included in the final analysis. Forty-one cases of NSCLC were localized on the left side and 49 on the right side, which included, respectively, 21 and 20 cases localized on the upper and lower lobe on the left side and 27, 8 and 14 cases localized on the upper, middle and lower lobe on the right side. Additionally, this group of patients included 72 (80.0\%) males and $18(20.0 \%)$ females, with a median age of 59 years (range, 28-77, IQR 55-65). Seventy-eight patients (86.7\%) were $<70$ years of age and $12(13.3 \%)$ were $\geq 70$ years. Fifty-one patients were never-smokers and 39 patients were current or 
Table I. Demographic and clinical features of the 90 NSCLC patients.

\begin{tabular}{ll}
\hline Characteristics $\quad$ No. of patients (\%) \\
\hline
\end{tabular}

Age (years)
Median (IQR)
$\geq 70$
$<70$

$59(55-65)$

$12(13.3)$

$78(86.7)$

Gender

Male

Female

Smoking history

Never-smokers

Current or ever smokers

Cell type

Squamous cell carcinoma

$41(45.6)$

Adenocarcinoma

$32(35.6)$

Adenosquamous carcinoma

$16(17.8)$

Others

Differentiation

Well

$16(17.8)$

Moderate

$49(54.4)$

Poor

$9(10.0)$

Missing data

$16(17.8)$

Tumor diameter $(\mathrm{cm})$

$<3$

$29(32.2)$

$\geq 3$

$61(67.8)$

Mediastinal lymph nodes

Positive

$34(37.8)$

Negative

$56(62.2)$

Stage

Ia

$\mathrm{Ib}$

$31(34.4)$

IIa

$12(13.3)$

$6 \quad(6.7)$

$\mathrm{IIb}$

$28(31.1)$

$1(1.1)$

5 (5.6)

IV

IHC for p53, EGFR, Kras $^{\mathrm{a}}$

$\begin{array}{ll}\text { p53(+) } & 45(54.2) \\ \operatorname{EGFR}(+) & 37(44.6) \\ \text { Kras(+) } & 41(49.4) \\ \text { Ki-67(+) } & 63(75.9)\end{array}$

aIHC for p53, EGFR, Kras, Ki-67 was carried out in 83 cases, while data were missing in 7 cases.

ever-smokers. Coexisting diseases, including hypertension, coronary heart disease, diabetes, obstructive pneumonia, gastric ulcer and varicose vein, were reported in 24 patients.
Table II. Distribution of tumor localization.

\begin{tabular}{lc}
\hline Localization & No. of patients (\%) \\
\hline Right lung & 49 \\
Upper lobe & $27(30)$ \\
Middle lobe & $8(8.9)$ \\
Lower lobe & $14(15.6)$ \\
Left lung & 41 \\
Upper lobe & $21(23.3)$ \\
Lower lobe & $20(22.2)$ \\
\hline
\end{tabular}

Ten patients underwent pneumonectomies, 66 lobectomies, and 6 bilobectomies. Lobectomy combined with extended resection was performed in 8 cases (4 cases of sleeve resection, 1 case of angioplasty and 3 cases of tracheoplasty). Postoperative complications developed in 14 cases and included prolonged air leakage ( $>1$ week in 8 cases), pulmonary infection (1 case), empyema (1 case), atrial fibrillation, requiring intervention (1 case), chylothorax (2 cases, requiring further operation in 1 case) and acute pulmonary embolism (1 case).

Pathology and immunohistochemistry. There were 41 (45.6\%) squamous cell carcinoma, 32 (35.6\%) adenocarcinoma, 16 $(17.8 \%)$ adenosquamous carcinoma and $1(1.1 \%)$ large-cell carcinoma cases in this group. Thirty-eight were stage PI (7 Ia and 31 Ib), 18 PII (12 IIa and 6 IIb), 29 PIII (28 IIIa and 1 IIIb) and 5 PIV. The reasons for PIIIb was metastatic isolate nodules in different lobes. The median tumor size was $3.5 \mathrm{~cm}$ (IQR $2.5-5 \mathrm{~cm}$, range $1.2-9.5 \mathrm{~cm}$ ). For tumor grading, well, moderately and poorly differentiated carcinoma was noted in 16, 49 and 9 cases, respectively (16 missing cases were adenosquamous carcinoma, for which the differentiation degree was not able to be distinguished). A total of 1268 lymph nodes (1045 mediastinal and 223 regional LNs) were removed at an average of $14 \pm 6.9$ nodes per patient. One hundred and eighty-five N2 nodes in 34 cases proved cancerous among the total LNs removed. The results of the IHC analysis were obtained from 83 cases. Following IHC analysis, positive expression of p53, EGFR, Kras and Ki-67 was observed in 45, 37, 41 and 63 specimens, respectively. Clinical characteristics of the 90 patients are shown in Table I; tumor localization is shown in Table II.

miR-150 and miR-3940-5p expression was reduced in tumor tissues and tumor-adjacent tissues compared to normal tissues. The expression of miR-150, miR-18b-5p, miR-643, miR-3940-5p and internal control snRNA U6 was detected by miRNA poly(A) tailing-based SYBR-Green real-time qPCR. The specificity of each miRNA primer for amplification was confirmed by a melting curve analysis at the end of the PCR procedure and subsequential agarose gel electrophoresis of the PCR products (Fig. 1).

We examined the expression levels of miR-150, miR-18b-5p, miR-643 and miR-3940-5p in the tumor, tumor-adjacent and normal tissues of the 90 NSCLC cases. As a result, a marked downregulation of miR-150 and miR-3940-5p expression was 

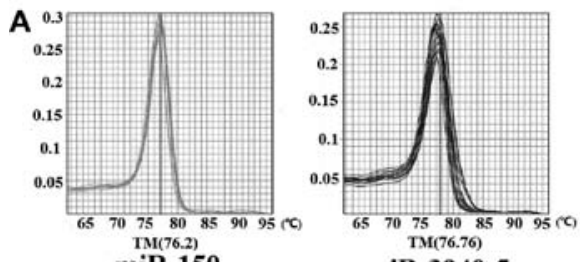

miR-150

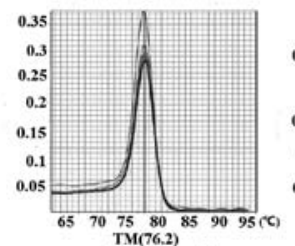

miR-18b-5p

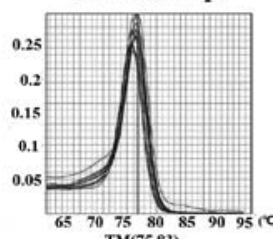

snRNAU6
B

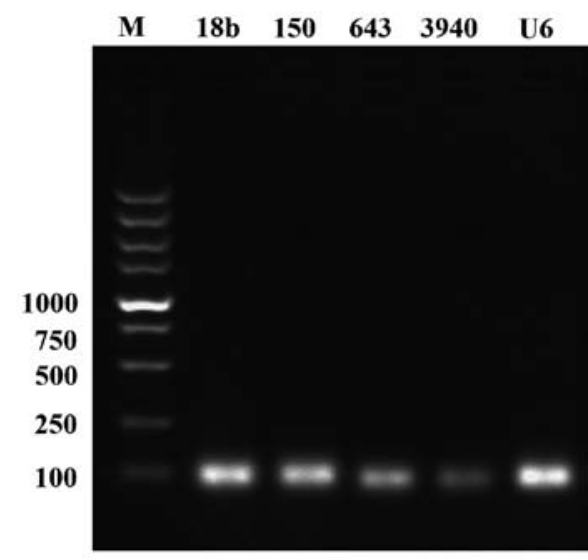

Figure 1. Specific amplification of miR-150, miR-3940-5p, miR-18b-5p and miR-643 by poly(A) tailing-based SYBR-Green real-time qPCR. (A) Melting curve analysis of miR-150, miR-3940-5p, miR-18b-5p, miR-643 and snRNA U6 demonstrates single peak. (B) Agarose gel electrophoresis of the PCR products demonstrates a single band.
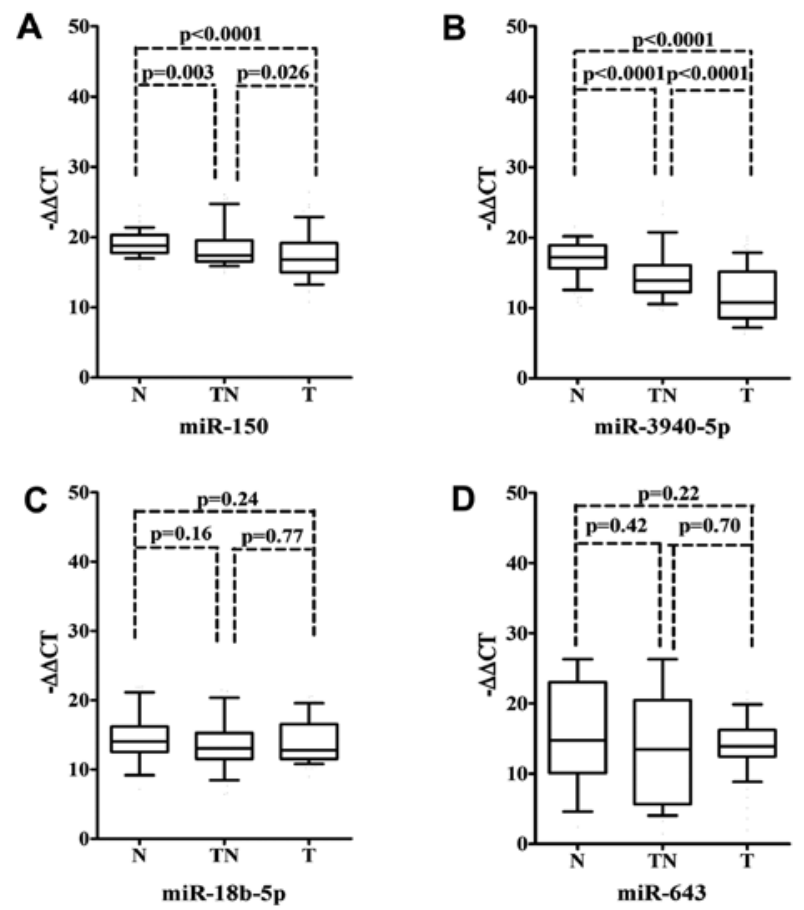

Figure 2. Expression of (A) miR-150, (B) miR-3940-5p, (C) miR-18b-5p and (D) miR-643 in normal, tumor-adjacent, and tumor tissues of 90 matched NSCLC samples. Expression levels of miR-150 and miR-3940-5p were significantly downregulated in tumor and tumor-adjacent tissues when compared to normal tissues. Expression levels of miR-150 and miR-3940-5p were also significantly downregulated in tumor compared to tumor adjacent tissues (N, normal; TN, tumor-adjacent normal; $\mathrm{T}$, tumor). The data were analyzed using Wilcoxon Mann-Whitney test.

noted in the tumor tissues when compared to the normal and tumor-adjacent tissues $(\mathrm{P}<0.05)$. The expression of miR-150 and miR-3940-5p in tumor-adjacent tissues was also lower
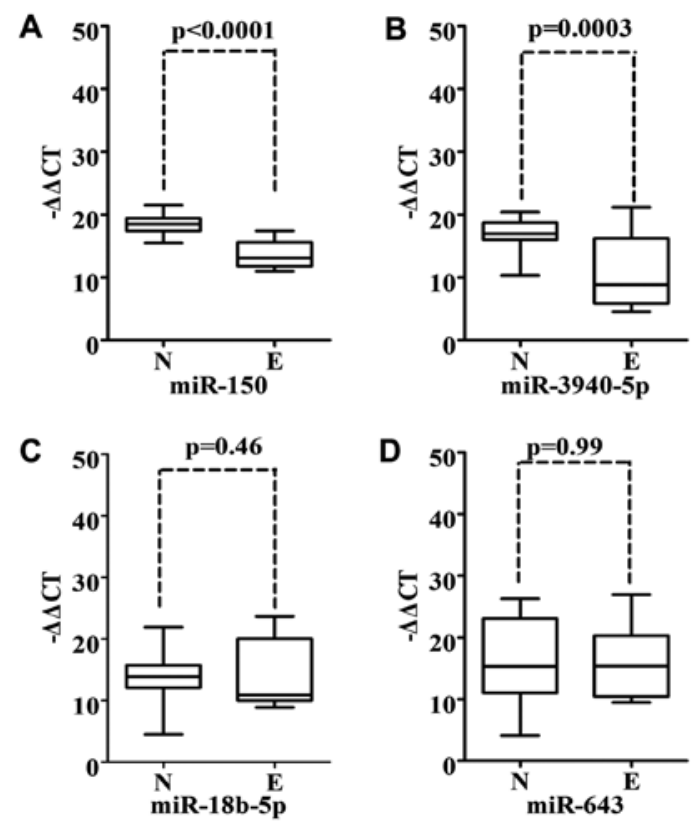

Figure 3. Expression of (A) miR-150, (B) miR-3940-5p, (C) miR-18b-5p and (D) miR-643 in normal and embryonic lung tissues. Expression of miR-150 and miR-3940-5p was significantly downregulated in the embryonic lung tissue (N, normal; E, embryonic).

than that in the the matched normal tissues $(\mathrm{P}<0.05)$ (Fig. $2 \mathrm{~A}$ and B). As for miR-18b-5p and miR-643, no significant differences were found among the groups $(\mathrm{P}>0.05)$ (Fig. 2C and D).

miR-150 and miR-3940-5p expression is reduced in embryonic lung tissue when compared to normal adult lung tissue. It has been reported that miRNAs are essential for regulating cell differentiation and maintaining the pluripotent state of stem cells (12). Overlapping miRNA expression patterns may exist 
Table III. Relationship between clinicopathological features and miR-150 and miR-3940-5p expression.

\begin{tabular}{|c|c|c|c|c|c|}
\hline & \multirow[b]{2}{*}{ No. of samples } & \multicolumn{2}{|c|}{$\operatorname{miR}-150$} & \multicolumn{2}{|c|}{ miR-3940-5p } \\
\hline & & $-\Delta \Delta \mathrm{Ct}$ & P-value & $-\Delta \Delta \mathrm{Ct}$ & P-value \\
\hline Age (years) & 90 & & & & \\
\hline$\geq 70$ & 12 & $17.2(14.7-20.5)$ & 0.44 & $17.2(14.7-20.5)$ & \\
\hline$<70$ & 78 & $16.5(15.9-19.1)$ & & $16.5(15.9-19.1)$ & 0.38 \\
\hline Gender & 90 & & & & \\
\hline Male & 72 & $16.77(14.91-19.06)$ & & $10.46(8.29-14.98)$ & \\
\hline Female & 18 & $16.85(15.44-20.11)$ & 0.68 & $12.25(8.28-17.07)$ & 0.53 \\
\hline Smoking habit & 90 & & & & \\
\hline Never-smokers & 51 & $17.69(16.35-20.29)$ & & $9.80(8.06-15.09)$ & \\
\hline Current or ever smokers & 39 & $16.05(14.56-18.70)$ & $0.03^{\mathrm{c}}$ & $12.21(9.26-15.24)$ & 0.18 \\
\hline Histological subtype & $89^{\mathrm{a}}$ & & & & \\
\hline Squamous cell & 41 & $16.46(14.85-20.17)$ & 0.07 & $10.75(8.19-15.26)$ & 0.72 \\
\hline Adenocarcinoma & 32 & $18.07(15.46-21.84)$ & & $12.61(8.60-16.82)$ & \\
\hline Adenosquamous & 16 & $16.11(14.45-17.13)$ & & $9.75(8.18-12.03)$ & \\
\hline Stage & 90 & & & & \\
\hline $\mathrm{I}+\mathrm{II}$ & 56 & $17.41(15.42-20.29)$ & $0.04^{\mathrm{c}}$ & $10.39(8.98-15.21)$ & 0.34 \\
\hline III-IV & 34 & $16.08(14.67-18.10)$ & & $10.34(7.81-14.83)$ & \\
\hline Differentiation & $74^{b}$ & & & & \\
\hline Well & 16 & $17.94(16.53-19.43)$ & 0.36 & $11.52(8.60-15.85)$ & 0.44 \\
\hline Moderate & 49 & $16.68(14.67-20.31)$ & & $12.41(8.67-15.28)$ & \\
\hline Poor & 9 & $15.88(14.38-20.65)$ & & $8.84(7.18-15.72)$ & \\
\hline Tumor diameter $(\mathrm{cm})$ & 90 & & & & \\
\hline$<3$ & 29 & $18.07(16.30-21.07)$ & $0.007^{\mathrm{c}}$ & $10.17(8.07-14.94)$ & 0.20 \\
\hline$\geq 3$ & 61 & $16.35(14.50-18.63)$ & & $12.61(8.81-15.63)$ & \\
\hline MLN & 90 & & & & \\
\hline Metastasis (+) & 34 & $16.32(14.78-18.24)$ & 0.25 & $11.04(8.84-15.26)$ & 0.47 \\
\hline No metastasis (-) & 56 & $17.05(14.95-20.29)$ & & $10.06(8.42-15.19)$ & \\
\hline
\end{tabular}

${ }^{\mathrm{a}}$ One case of large cell carcinoma was excluded. ${ }^{\mathrm{b}}$ Sixteen missing cases were of adenosquamous carcinoma, for which the degree of differentiation was not able to be distinguished. ${ }^{c}$ Wilcoxon Mann-Whitney test for two groups; Kruskal-Wallis H test for several independent samples. MLN, mediastinal lymph node.

between tumor and embryonic cells (13). Therefore, we examined the expression of miR-150, miR-3940-5p, miR-18b-5p and miR-643 in 17 cDNA samples of embryonic lung tissue. Notably, we observed that the expression of miR-150 and miR-3940-5p was significantly downregulated in embryonic lung tissue vs. normal adult lung tissue $(\mathrm{P}<0.001)$ (Fig. 3A and 3B). We did not note different expression levels of miR-18b-5p and miR-643 in embryonic lung tissue when compared to the normal adult lung tissue (Fig. 3C and D).

Relationship between clinicopathological features and miR-150 and miR-3940-5p expression levels. The expression levels of miR-150 and miR-3940-5p were compared between different cohorts dependent on various clinicopathological features (Table III). There were no significant variations in miR-150 between the subgroups regarding age, gender, tumor differentiation and metastasis to mediastinal lymph nodes. However, a statistically significant difference in miR-150 expression was observed in regards to tumor size, smoking and stage. The expression level of miR-150 was much lower in the subgroups of smokers and patients with tumors of III-IV stage and a diameter $\geq 3 \mathrm{~cm}$. A significant difference in miR-3940-5p expression was not noted between subgroups according to age, gender, tumor differentiation, smoking status, histology, tumor stage, tumor size and metastasis to mediastinal lymph nodes.

Low expression of miR-150 and miR-3940-5p is associated with the Ki-67 proliferation index in NSCLC. The percentage of $\mathrm{Ki}$-67-positive tumor cells in a tumor is an excellent marker of tumor proliferation, and is correlated with prognosis for survival and tumor recurrence. After designating patients with a Ki-67 labeling index $>5 \%$ as the positive group, we found that miR-150 and miR-3940-5p expression levels were specifically downregulated in the Ki-67-positive NSCLC group, while miR-18b-5p and miR-643 expression levels were not altered significantly between the Ki-67-positive and -negative NSCLC groups (Fig. 4). This finding implies that miR-150 and 
Table IV. miR-150 and miR-3940-5p expression in p53, EGFR, Kras IHC-positive and -negative NSCLC cases.

\begin{tabular}{|c|c|c|c|c|c|}
\hline \multirow[b]{2}{*}{ Protein expression } & \multirow[b]{2}{*}{ No of samples } & \multicolumn{2}{|c|}{$\operatorname{miR}-150$} & \multicolumn{2}{|c|}{ miR-3940-5p } \\
\hline & & $-\Delta \Delta \mathrm{Ct}$ & P-value & $-\Delta \Delta \mathrm{Ct}$ & $\mathrm{P}$-value \\
\hline p53 & 83 & & & & \\
\hline$(+)$ & 45 & $16.50(15.36-18.19)$ & $0.04^{\mathrm{a}}$ & $10.01(8.19-15.01)$ & $0.04^{\mathrm{a}}$ \\
\hline$(-)$ & 38 & $18.19(15.46-21.88)$ & & $13.73(9.48-15.95)$ & \\
\hline EGFR & 83 & & & & \\
\hline$(+)$ & 37 & $16.45(14.58-20.29)$ & 0.34 & $12.21(9.01-16.02)$ & 0.49 \\
\hline$(-)$ & 46 & $17.70(15.50-19.13)$ & & $10.35(8.71-15.10)$ & \\
\hline Kras & 83 & & & & \\
\hline$(+)$ & 41 & $16.77(14.88-19.11)$ & 0.25 & $10.04(8.07-15.20)$ & 0.12 \\
\hline$(-)$ & 42 & $17.25(15.89-20.27)$ & & $12.21(9.40-15.58)$ & \\
\hline
\end{tabular}

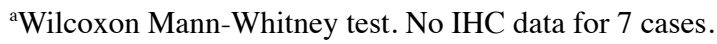

A

ADC SQC AD-SQC

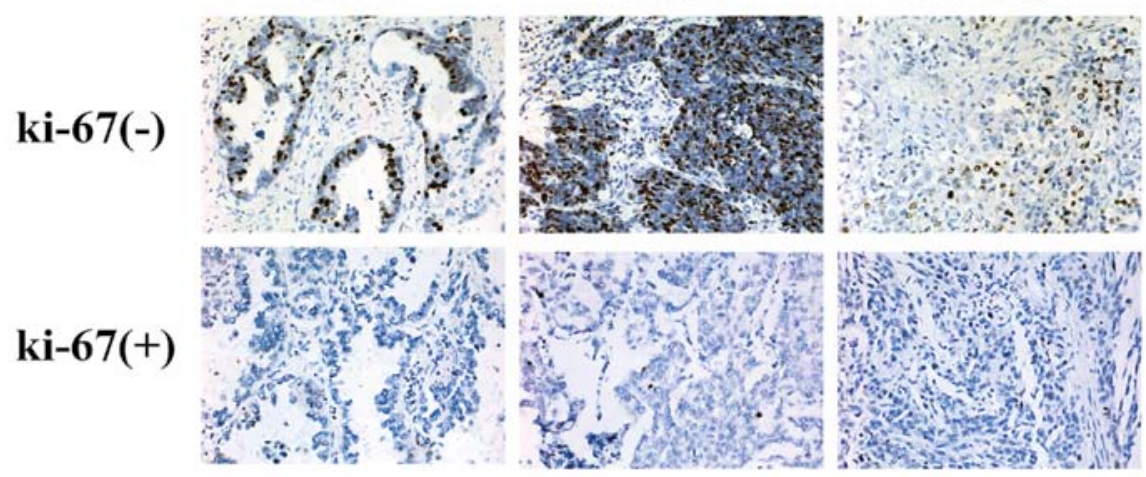

B
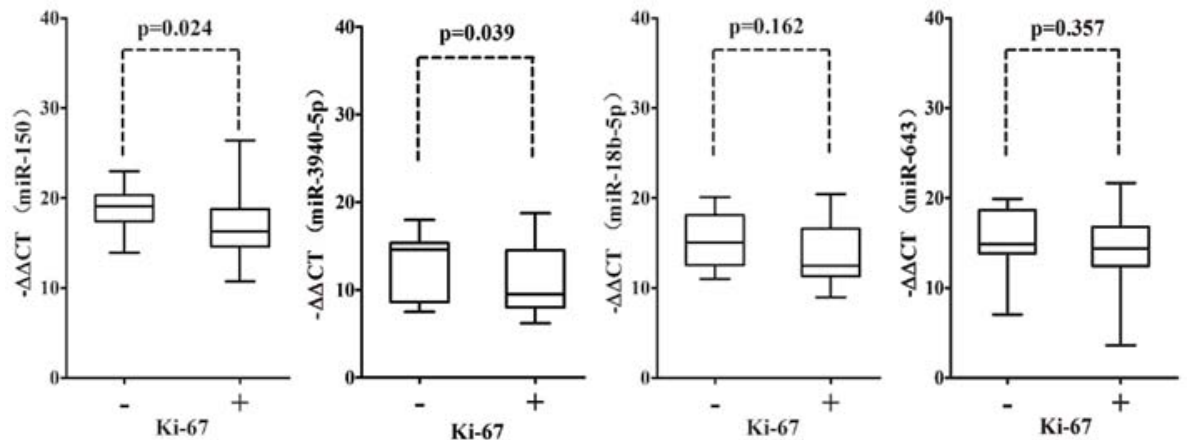

Figure 4. Low expression of miR-150 and miR-3940-5p is associated with the Ki-67 proliferation index in NSCLC. (A) Representative images of Ki-67 IHCpositive and -negative NSCLC (ADC, adenocarcinoma; SQC, squamous carcinoma; AD-SQC, adenosquamous carcinoma). (B) miR-150 and miR-3940-5p expression levels were significantly downregulated in the Ki-67-positive NSCLC cases ( $\mathrm{n}=83$, Wilcoxon Mann-Whitney test).

miR-3940-5p expression may be associated with tumor growth and proliferation in NSCLC.

Correlation between expression levels of miR-150, miR-3940-5p and tumor-related genes Kras, EGFR, p53. As shown in Table IV and Fig. 5A, we analyzed the miR-150 and miR-3940-5p expression levels in 83 cases of NSCLC in subgroups categorized according to positive or negative IHC staining for tumor-related genes p53, Kras and EGFR. The levels of miR-150 and miR-3940-5p expression were significantly downregulated in the p53 IHC-positive NSCLC, but not in the Kras- and EGFR-positive NSCLC cases. To confirm the possible p53 association with miR-150 and miR-3940-5p, we also examined the p53 mRNA expression in 60 cases of paired NSCLC samples by quantitative real-time PCR. p53 mRNA was highly expressed in the tumor samples of NSCLC 
A

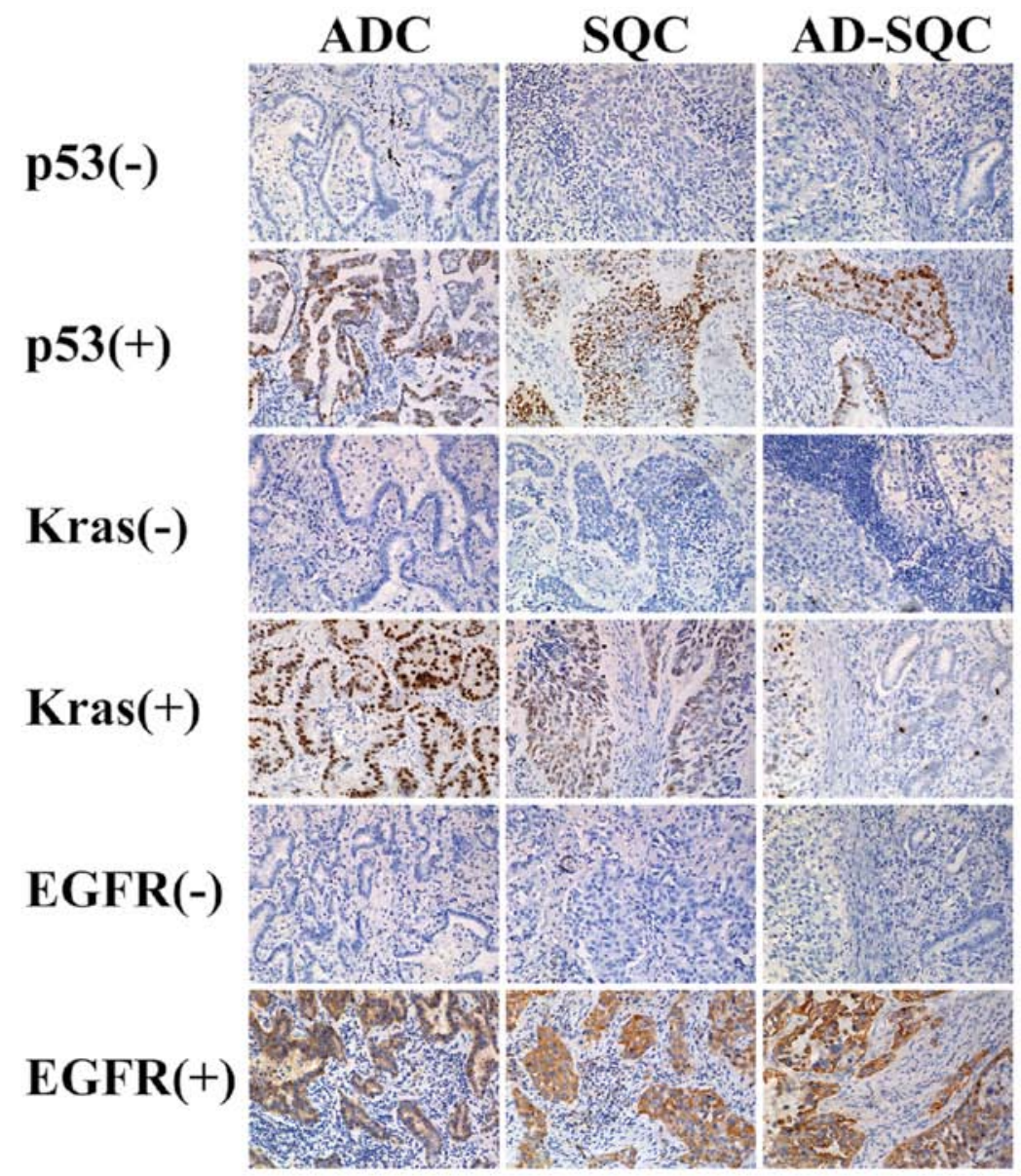

B

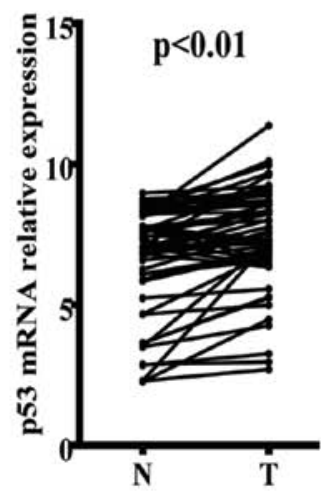

C

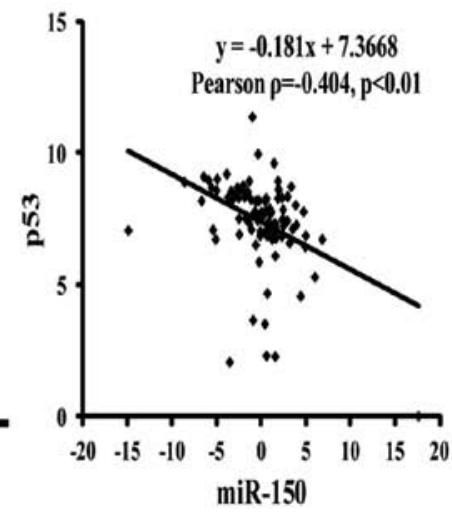

D

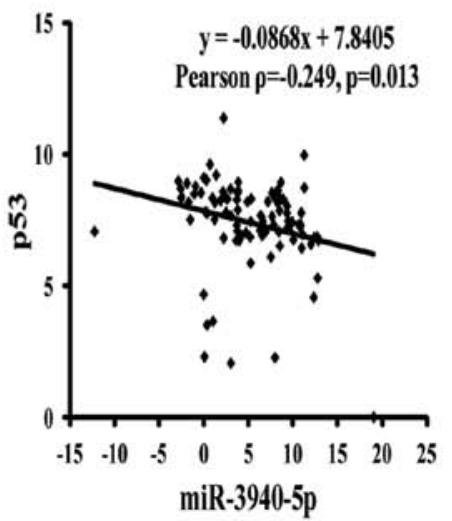

Figure 5. Correlation between miR-150, miR-3940-5p and tumor-related genes p53, Kras and EGFR. (A) Representative images of p53, Kras and EGFR IHC-positive and -negative NSCLC (magnification, x200). (B) p53 mRNA expression was significantly higher in NSCLC tumor tissues compared to matched normal tissues by quantitative real-time PCR ( $\mathrm{n}=60$, paired Student's t-test, $\mathrm{P}<0.01$ ). (C) Correlation between $\mathrm{p} 53 \mathrm{mRNA}$ expression level and miR-150 expression in NSCLC ( $\mathrm{n}=120,60$ for tumor and 60 for normal; Pearson's correlation). (D) Correlation between p53 mRNA expression level and miR-3940-5p expression in NSCLC ( $\mathrm{n}=120,60$ for tumor and 60 for normal; Pearson's correlation).

(Fig. 5B). Additionally, the p53 mRNA expression level was negatively correlated with miR-150 and miR-3940-5p (Fig. 5C) expression in the normal and tumor tissues of NSCLC.

\section{Discussion}

The present study investigated the expression pattern of miR-150 and miR-3940-5p in lung cancers. Specifically, this investigation yielded the following findings. i) A significant decrease in the expression levels of miR-150 and miR-3940-5p were noted in the tumor tissues when compared to normal tissues and tumor-adjacent tissues vs. normal tissues. ii) Downregulated expression of miR-150 and miR-3940-5p was found in embryonic lung tissue. iii) miR-150 expression was associated with smoking in NSCLC patients; decreased miR-150 expression in tumor tissues was found in a cohort of 
smokers. iv) miR-150 was preferentially downregulated in a subgroup of NSCLC patients with a tumor diameter $\geq 3 \mathrm{~cm}$. v) miR-150 was downregulated in stages III and IV compared to stages I and II tumors. vi) miR-150 and miR-3940-5p were most aberrantly decreased in nuclear proliferating antigen Ki-67-positive NSCLC. vii) miR-150 and miR-3940-5p were expressed to a lesser degree in p53 IHC-positive NSCLC. viii) Expression levels of miR-150 and miR-3940-5p were negatively correlated with p53 mRNA in NSCLC. There was no significant difference in the expression level of miR-150 in regards to age, tumor histology and differentiation (Table III). These findings demonstrate that miR-150 and miR-3940-5p may play a tumor-suppressing role in lung carcinogenesis.

The present study showed that nuclear positive $\mathrm{Ki}-67$ of NSCLC is associated with miR-150 and miR-3940-5p downregulation. $\mathrm{Ki}-67$ is commonly used as a marker to evaluate proliferation of tumor cells. It is strictly associated with cell proliferation and is present during all active phases of the cell cycle, but is absent in resting cells (14). Findings convincingly support that $\mathrm{Ki}-67$ overexpression in resected NSCLC is associated with a poor prognosis (15-17). Downregulation of miR-150 and miR-3940-5p in Ki-67-positive specimens suggests that miR-150 and miR-3940-5p may be involved in tumor cell proliferation and contribute to poor prognosis of NSCLC.

miR-150 has been reported as a hematopoietic-specific miRNA in malignant lymphoma and is significantly downregulated in tumor cells relative to healthy cells (18). In solid tumor tissue, miR-150 expression levels were reduced when compared to paired non-cancerous tissue in colorectal cancer, indicating that low miR-150 expression is associated with shorter survival and a worse response to adjuvant chemotherapy (19). Our results demonstrated that miR-150 expression is also reduced in another solid tumor - NSCLC. Additionally, we found that miR-150 expression was strongly associated with tumor stage and size as significantly low expression in advanced-stage and large-size tumors was noted. Moreover, we obtained significant findings concerning the effect of tobacco smoking on miR-150 expression; the expression of miR-150 was much lower in the tumors of smokers than that in non-smokers. It has been observed that smoking status may have differential impacts on miRNA expression for mutational patterns of EGFR in NSCLC (20), and downregulation of let-7 associated with tobacco smoking was also observed in rats (21). Modification of miR-150 expression in our research suggests that smoking status has a negative effect on the potential tumor-suppressor miR-150 in NSCLC. However, we did not conduct a hierarchical analysis on the effect of cigarettes because of the small number of cases. Other unknown factors may have contributed to our results; therefore, larger samples or functional tests are necessary to explicate our results.

miR-3940-5p is located at chr-19 p13.3, and the 5p-maturesequence is GUGGGUUGGGGCGGGCUCUG; this is a novel miRNA and was first reported in human embryonic stem cells (candidate-18) (22). Next-generation sequencing identified a large number of new miRNAs, including miR-3940-5p. These new miRNAs are generally less evolutionarily conserved than known miRNAs, with a large percentage unique to humans or primates; the importance of non-conserved miRNAs has not been fully appreciated (23). To date, there is no report on the expression level and regulation of miR-3940-5p in any cell line or solid tumor. The results of our study found that miR-3940-5p was downregulated in NSCLC and embryonic lung tissue and may have the potential function as a tumor suppressor in the carcinogenesis of lung cancer.

Our findings also demonstrated that miR-150 and miR-3940-5p expression was much lower in p53 IHC-positive NSCLC. p53 protein is expressed at low levels in normal cells and at high levels in a variety of transformed cell lines, where it is believed to contribute to transformation and malignancy (24). Nuclear p53 protein accumulation, in most cases, is due to mutations within the gene and a strong association was noted between TP53 mutations and nuclear p53 protein accumulation (25). Mutations in this gene and accumulation of the abnormal p53 protein are frequently associated with malignancy and tumor progression; accumulation of the mutant p53 protein has been associated with a poor prognosis in patients with breast, gastric and colorectal carcinomas (26). It is possible that miR-150 and miR-3940-5p directly or indirectly regulate the p53 expression or degradation. Regulation of p53 activity, by different miRNAs, has been verified in several pathways. For example, miR-34a acts as a negative regulator of p53 through deacetylating p53 (27); miR-29 family members upregulate p53 protein levels and induce p53-mediated apoptosis through direct downregulation of the regulatory subunit of PI3K-p85 $\alpha(28,29)$. miR-122 was found to modulate p53 through the inhibition of cyclin G1 (30). Prediction of miR-150 target gene by TargetScan 6.0 demonstrated that a conserved 8mer binding site of miR-150 exists at the 3'UTR of TP53/ p53. Thus, as a potential tumor suppressor, hsa-miR-150 may be involved in the alteration of p53 gene expression. Few studies have been conducted on the expression and function of hsa-miR-3940-5p in carcinoma. Our results suggest that hsamiR-3940-5p may also be involved in the regulation of p53 expression, directly or indirectly, but further elucidation of the underlying mechanism of decreased miR-3940-5p expression in 53-positive cases is needed.

We also examined the downregulation of miR-150 and miR-3940-5p in embryonic lung tissue compared to a normal adult sample. miRNAs play critical roles in the maintenance of stemness in ES cells as well as in the differentiation to multiple cell lineages (12). In previous reports, oncogenic miRNAs were upregulated in hES cells, which suggests a possible function in the blockade of cell differentiation $(31,32)$. Overexpression of let-7b led to reduced neural stem cell proliferation and increased neural differentiation (33), and inhibition of the let-7 family promotes de-differentiation of somatic cells to induce pluripotent stem (iPS) cells (34). It has been reported that the downregulation of let-7 is essential for self-renewal and maintenance of the undifferentiated state of cancer stem cells (35). Downregulation of miR-150 and miR-3940-5p in embryonic lung tissue implies that they can interfere with the differentiation of stem or carcinoma stem cells.

We found that miR-150 and miR-3940-5p were significantly differentially expressed between tumor, tumor-adjacent and normal tissues, and miR-150 was associated with tumor stage and size. This is evidenced by the significantly low expression in advanced-stage and large-size tumors. Notably, downregulated miR-150 was significantly associated with smoking status in NSCLC, which implies that miR-150 and miR-3940-5p are involved in the pathogenesis of NSCLCS. 
miR-150 and miR-3940-5p were also significantly downregulated in embryonic lung tissues compared to a normal sample, which suggests they may be involved in the differentiation of stem cells of normal or cancer cells. We also found that miR-150 and miR-3940-5p were specifically downregulated in nuclear cell proliferation antigen Ki-67-positive NSCLC, which suggests that miR-150 and miR-3940-5p may be involved in tumor cell proliferation and may contribute to poor prognosis. miR-150 and miR-3940-5p were significantly downregulated in p53 IHC-positive cases, but not in Krasand EGFR-positive cases. We confirmed that miR-150 and miR-3940-5p expression was negatively correlated with $\mathrm{p} 53$ mRNA by real-time PCR in NSCLC. These findings suggest that miR-150 and miR-3940-5p interfere in NSCLC tumorigenesis and progression, and in the direct or indirect pathways of miR-150 and miR-3940-5p to affect p53 expression. These findings warrant further research.

\section{Acknowledgements}

This study was supported by the Science and Technology Commission of Shanghai Municipality (10411956100), (12ZR1426100) and Wu Jieping Funding (320.6720.1003). We are extremely grateful to Mr. Liang Tang and Miss Xiaojun Yang for their experimental expertise and Dr Gang Chen for providing the resection specimens.

\section{References}

1. Bartel DP: MicroRNAs: genomics, biogenesis, mechanism, and function. Cell 116: 281-297, 2004.

2. Eder $M$ and Scherr M: MicroRNA and lung cancer. N Engl J Med 352: 2446-2448, 2005.

3. Scott GK, Goga A, Bhaumik D, Berger CE, Sullivan CS and Benz CC: Coordinate suppression of ERBB2 and ERBB3 by enforced expression of micro-RNA miR-125a or miR-125b. J Biol Chem 282: 1479-1486, 2007.

4. Calin GA and Croce CM: MicroRNA signatures in human cancers. Nat Rev Cancer 6: 857-866, 2006.

5. Jemal A, Siegel R, Ward E, Murray T, Xu J and Thun MJ: Cancer statistics, 2007. CA Cancer J Clin 57: 43-66, 2007.

6. Goldstraw P, Crowley J, Chansky K, et al: The IASLC Lung Cancer Staging Project: proposals for the revision of the TNM stage groupings in the forthcoming (seventh) edition of the TNM Classification of Malignant Tumours. J Thorac Oncol 2: 706-714, 2007.

7. Govindan R, Page N, Morgensztern D, et al: Changing epidemiology of small-cell lung cancer in the United States over the last 30 years: analysis of the surveillance, epidemiologic, and end results database. J Clin Oncol 24: 4539-4544, 2006.

8. Takamizawa J, Konishi H, Yanagisawa K, et al: Reduced expression of the let-7 microRNAs in human lung cancers in association with shortened postoperative survival. Cancer Res 64: 3753-3756, 2004.

9. Yu SL, Chen HY, Chang GC, et al: MicroRNA signature predicts survival and relapse in lung cancer. Cancer Cell 13: 48-57, 2008.

10. Raponi M, Dossey L, Jatkoe T, et al: MicroRNA classifiers for predicting prognosis of squamous cell lung cancer. Cancer Res 69: 5776-5783, 2009.

11. Soomro IN, Holmes J and Whimster WF: Predicting prognosis in lung cancer: use of proliferation marker, Ki67 monoclonal antibody. J Pak Med Assoc 48: 66-69, 1998.

12. Tiscornia G and Izpisua Belmonte JC: MicroRNAs in embryonic stem cell function and fate. Genes Dev 24: 2732-2741, 2010.
13. Monzo M, Navarro A, Bandres E, et al: Overlapping expression of microRNAs in human embryonic colon and colorectal cancer. Cell Res 18: 823-833, 2008.

14. Scholzen T and Gerdes J: The Ki-67 protein: from the known and the unknown. J Cell Physiol 182: 311-322, 2000.

15. Takahashi S, Kamata Y, Tamo W, et al: Relationship between postoperative recurrence and expression of cyclin E, p27, and $\mathrm{Ki}-67$ in non-small cell lung cancer without lymph node metastases. Int J Clin Oncol 7: 349-355, 2002.

16. Martin B, Paesmans M, Mascaux C, et al: Ki-67 expression and patients survival in lung cancer: systematic review of the literature with meta-analysis. Br J Cancer 91: 2018-2025, 2004.

17. Woo T, Okudela K, Yazawa T, et al: Prognostic value of KRAS mutations and $\mathrm{Ki}-67$ expression in stage I lung adenocarcinomas. Lung Cancer 65: 355-362, 2009.

18. Watanabe A, Tagawa H, Yamashita J, et al: The role of microRNA150 as a tumor suppressor in malignant lymphoma. Leukemia 25: $1324-1334,2011$

19. Ma Y, Zhang P, Wang F, et al: miR-150 as a potential biomarker associated with prognosis and therapeutic outcome in colorectal cancer. Gut 61: 1447-1453, 2011.

20. Toyooka S, Matsuo K, Shigematsu H, et al: The impact of sex and smoking status on the mutational spectrum of epidermal growth factor receptor gene in non-small cell lung cancer. Clin Cancer Res 13: 5763-5768, 2007.

21. Izzotti A, Calin GA, Arrigo P, Steele VE, Croce CM and De Flora S: Downregulation of microRNA expression in the lungs of rats exposed to cigarette smoke. FASEB J 23: 806-812, 2009.

22. Liao JY, Ma LM, Guo YH, et al: Deep sequencing of human nuclear and cytoplasmic small RNAs reveals an unexpectedly complex subcellular distribution of miRNAs and tRNA 3 trailers. PLoS One 5: e10563, 2010.

23. Persson H, Kvist A, Rego N, et al: Identification of new microRNAs in paired normal and tumor breast tissue suggests a dual role for the ERBB2/Her2 gene. Cancer Res 71: 78-86, 2011.

24. Starzynska T, Bromley M, Ghosh A and Stern PL: Prognostic significance of p53 overexpression in gastric and colorectal carcinoma. Br J Cancer 66: 558-562, 1992.

25. Andersen TI, Holm R, Nesland JM, Heimdal KR, Ottestad L and Borresen AL: Prognostic significance of TP53 alterations in breast carcinoma. Br J Cancer 68: 540-548, 1993.

26. Chava S, Mohan V, Shetty PJ, et al: Immunohistochemical evaluation of p53, FHIT, and IGF2 gene expression in esophageal cancer. Dis Esophagus 25: 81-87, 2012.

27. Yamakuchi M, Ferlito $M$ and Lowenstein CJ: miR-34a repression of SIRT1 regulates apoptosis. Proc Natl Acad Sci USA 105: 13421-13426, 2008.

28. Park SY, Lee JH, Ha M, Nam JW and Kim VN: miR-29 miRNAs activate $\mathrm{p} 53$ by targeting $\mathrm{p} 85$ alpha and CDC42. Nat Struct Mol Biol 16: 23-29, 2009.

29. Zhou BP, Liao Y, Xia W, Zou Y, Spohn B and Hung MC: HER-2/ neu induces p53 ubiquitination via Akt-mediated MDM2 phosphorylation. Nat Cell Biol 3: 973-982, 2001.

30. Fornari F, Gramantieri L, Giovannini C, et al: MiR-122/cyclin G1 interaction modulates p53 activity and affects doxorubicin sensitivity of human hepatocarcinoma cells. Cancer Res 69: 5761-5767, 2009

31. Laurent LC, Chen J, Ulitsky I, et al: Comprehensive microRNA profiling reveals a unique human embryonic stem cell signature dominated by a single seed sequence. Stem Cells 26: 1506-1516, 2008.

32. Ren J, Jin P, Wang E, Marincola FM and Stroncek DF: MicroRNA and gene expression patterns in the differentiation of human embryonic stem cells. J Transl Med 7: 20, 2009.

33. Zhao C, Sun G, Li S, et al: MicroRNA let-7b regulates neural stem cell proliferation and differentiation by targeting nuclear receptor TLX signaling. Proc Natl Acad Sci USA 107: 1876-1881, 2010.

34. Melton C, Judson RL and Blelloch R: Opposing microRNA families regulate self-renewal in mouse embryonic stem cells. Nature 463: 621-626, 2010.

35. Yu F, Yao H, Zhu P, et al: let-7 regulates self renewal and tumorigenicity of breast cancer cells. Cell 131: 1109-1123, 2007. 